The Functioning of Economic Research; The Complexity of Economic Phenomena: R...

\title{
The Functioning of Economic Research
}

\author{
Jan Tinbergen
}

This note is the result of a promise to Professor Kurt Dopfer after a discussion we had last year [Dopfer 1988; Tinbergen, 1988]. It describes my way of conducting economic research, this being the subject of our discussion. In contrast to Professor Dopfer I purposely avoid the subject of mechanics and only deal with economics: I do not want to suggest $a$ priori a relation between these two sciences. To me the purpose of economic research is either (a) to attempt to explain an economic phenomenon or (b) to recommend an economic policy or structure. I will not use any philosophy of science concepts; I am not a philosopher and I think the treatment of my subject does not need these concepts, contrary to Professor Dopfer's opinion.

\section{Qualitative and Quantitative Phenomena}

An important characteristic of my views is that I deal with qualitative and quantitative aspects of economic research. Quantitative research must be preceded by qualitative research activities, if only to indicate the nature of things to be measured. Consequently, economic research cannot be quantitative alone. It may be of qualitative nature alone, however, if only qualitative phenomena play a role. One example is the proposition that there is an optimal level of decisionmaking to solve each problem (see Fischer and Tinbergen [1987]). I will address

The author is Emeritus Professor of Development Planning, Erasmus University of Rotterdam. 
that proposition in more detail later on. Other examples are definitions of concepts, for instance a definition of an institution. An institution may be defined as a group of human beings with certain tasks and the structure (infra- and superstructure) required for the execution of these tasks.

\section{Causality}

Crucial for scientific research is the concept of cause. A phenomenon $P_{1}$ is a cause of a phenomenon $P$ if the existence or change of $P$ always occurs after the coming into existence or change of $P_{1}$. A change may be qualitative (change in nature) or quantitative (change in size). The coming into existence or change of $P$ may occur after the coming into existence or change of $P_{1}, P_{2}, \ldots, P_{n}, \ldots P_{N}$. Then $P$ has several causes. A science is the set of causal connections among certain phenomena. Economic science deals with phenomena of a human population and the satisfaction this population derives from scarce goods and services and their transformation. Many phenomena with which economic science deals are quantitative. Consequently, for economic science, the solution of quantitative problems is important.

\section{Essential and Non-Essential Causes}

The causes of change in an economic phenomenon $P$ may be changes in explanatory phenomena (economic and other). The explanatory phenomena-the changes of which cause small changes in $P$ compared to other explanatory phenomena-are not essential to the explanation of $P$. Explanatory phenomena $P_{n}$, that cause a large change in $P$ compared to those caused by other $P_{\mathrm{n}}$ are essential. Non-essential changes in $P$ often are numerous and independent of each other and are often approached by random changes, a subject of the science of statistics. I shall not pursue this subject any further.

\section{Linearity of Relationships}

The relationship between a change of variable (the mathematical word to indicate a quantitative phenomenon) $P$ and the changes in some of its causes $\left(P_{\mathrm{n}}(n=1, \ldots, N)\right.$ may be linear but in most cases is curvilinear.

Over small intervals, most curvilinear relationships can be approximated by a linear relationship. For the solution of many problems this 
linearization constitutes an efficient technique: it reduces work without much loss in exactitude.

\section{Quantitative Problems and Their Solution}

A relevant part of economic science consists of problems in which only quantitative phenomena occur. Another part consists of problems in which only qualitative phenomena occur. Third, in some problems both qualitative and quantitative phenomena occur. In this section I will discuss the first type of problems and their solution.

If a set of relationships between a set of variables exists, that set of relationships or of equations (as mathematicians call them) can be used to solve a number of problems. We call this set of equations a model. In each problem some phenomena are supposed to be known and others are supposed to be unknown. The solution of a problem consists of determining the numerical values of the unknown variables. The variables occurring in a model have different natures. We distinguish target variables (or targets), instrument variables (or instruments), data variables (or data) and other variables, which are those left over. The coefficients in the equations characterize the structure of the economy described by the model.

A model can be used to solve explanatory problems (positive economic science) or to solve forecasting, policy, or planning problems (applied economic science). In explanatory problems the unknowns are target and other variables; instrument and data variables are known. In forecasting, policy, and planning problems, instrument and other variables are unknown; target and data variables are known. The same model can be used to solve both types of problems.

To solve a problem the number of equations has to be equal to the number of unknowns. It follows that the number of instruments must be equal to the number of targets. ${ }^{1}$ Exceptions to this rule are possible only if the structure of the equations is abnormal.

In economic research where measurable phenomena are involved, such measurement must be part of the research.

\section{Qualitative Problems and Their Solution}

Qualitative phenomena do not have a size. Only their nature can be described. They may exist or not exist, and may exist in a finite number of categories. they may be created by human beings with a certain purpose, as is an institution (see above). They may have a certain structure 
and may have a number of dimensions, such as the concept of security (see Fischer and Tinbergen [1987]). Their structure may consist of elements that can be ordered.

An example of a qualitative phenomenon is the decisionmaking structure addressing problems of general interest within all nations (see Section 2). Let us consider a nation called $\bar{N}_{n}$, where the index $n$ is the number given to that nation. If we number all nations of the world, starting with the nation with the largest population, $n=1$ stands for China, $n=2$ for India, et cetera. The bar indicates that the symbol stands for the nation's name; $N$ without a bar is the highest value of $n$, somewhat higher than 160 . (The number of members of the United Nations is 159). Let the nation considered consist of $P_{\mathrm{n}}$ provinces, numbered $p_{\mathrm{n}}$, with the names $\bar{P}_{\mathrm{pn}}$. For the Netherlands, $P_{\mathrm{n}}=12$, for Belgium, $P_{\mathrm{n}}=9$. Let the provinces be subdivided into $M_{\mathrm{pn}}$ municipalities, named $\bar{M}_{\text {mpn }}$ and ordered from the municipality with the highest number of inhabitants, then $m_{\mathrm{pn}}=1$ stands for Rotterdam, $m_{\mathrm{pn}}=3$ for The Hague. Each municipality may be subdivided into districts named $\bar{D}_{\text {mpn }}$ and numbered $d_{\mathrm{mpp}}$. Finally we consider as the lowest level of decisionmaking the household level $h_{\text {dmpn }}$, of which in the Netherlands the total number $H_{\text {dmpn }}=5.522$ million (in 1985). All the higher levels are those of representatives or authorities of groups of households, but only the public groups, not such private groups as associations, firms, churches, the association of municipalities. ble I.

The phenomenon of the decisionmaking structure is described in Ta-

Table 1. The national decision-making structure.

\begin{tabular}{lllc}
\hline Area & Name & \multicolumn{1}{c}{ Numbering } & $\begin{array}{c}\text { Level of } \\
\text { decisionmaking }\end{array}$ \\
\hline Nation & $\bar{N}_{n}$ & $n=1 \ldots N$ & $n$ \\
Province & $\bar{P}_{p n}$ & $p_{n}=1 \ldots P_{n}$ & $p_{n}$ \\
Municipality & $\bar{M}_{m p n}$ & $m_{p n}=1 \ldots M_{p n}$ & $m_{p n}$ \\
District & $\bar{D}_{d m p n}$ & $d_{m p n}=1 \ldots D_{m p n}$ & $d_{m p n}$ \\
Household & $\bar{H}_{h d m p n}$ & $h_{d m p n}=1 \ldots H_{d m p n}$ & $h_{d m p n}$ \\
\hline
\end{tabular}

A qualitative problem that can be solved with the aid of Table $I$ is the problem of the optimal decisionmaking level for a given problem. Any socio-economic problem can be characterized, to begin with, by the households or group of households whose welfare is affected. Thus, the problem of what food will be consumed on June 1, 1989 by household $\bar{H}_{14532}$ only affects that household. Or, the problem of adding a 
new tram route to the existing network in The Hague municipality affects the welfare of a number of households in a number of districts of that municipality. The lowest level of decisionmaking where the interests of these households are represented is the level of The Hague municipality. Strictly speaking a number of households living elsewhere also have an interest in the decision on that route, but their number is negligable in comparison to the number of interested citizens in The Hague.

The solution of the problem must satisfy two conditions: the optimal level of decisionmaking must (a) be as low as possible, since that means the most democratic solution and, in all likelihood the level where a maximum of knowledge is available, ${ }^{2}$ and (b) be that where all citizens whose interests are involved are represented. Table II illustrates the solution.

Table 2. Illustration of the solution of the problem of the optimal level of decisionmaking.

\begin{tabular}{cc}
\hline $\begin{array}{c}\text { Level where all interests } \\
\text { involved are represented }\end{array}$ & $\begin{array}{c}\text { Lowest of } \\
\text { these levels }\end{array}$ \\
\hline \hline$h d m p n$ & $h$ \\
$d m p n$ & $d$ \\
$m p n$ & $m$ \\
$p n$ & $p$ \\
$n$ & $n$ \\
\hline
\end{tabular}

The theorem of the Optimal Decisionmaking Level constitutes an example of solving a qualitative problem. The tools used here are not those of algebra, but the tools of definition, ordering, selecting, and checking or verification. Its importance can be shown by problems where (as in environmental policy) the welfare of various nations is involved. Then the optimal level is supranational and a new authority may have to be created.

The difference between quantitative and qualitative problems is that the former are solved completely only after measurement and mathematical solution with the aid of a model, whereas the latter do not require the tools of measurement and mathematical solution.

\section{Notes}

1. Let the number of targets be $t$, the number of instruments $i$, the number of data variables $d$, the number of other variables $o$, and the number of equations $e$, then $e=t+o$ and $e=i+o$. It follows that $t=i$. 
2. Knowledge of very complicated problems may be accumulated elsewhere but, as a rule, will be made available by contract. If not, the solution is different.

\section{References}

Dopfer, K. 1988. "Classical Mechanics With an Ethical Dimension: Professor Tinbergen's Economics." Journal of Economic Issues 22 (September): 675706.

Fischer, D. and J. Tinbergen, 1987. Warfare and Welfare, Brighton: Wheatsheaf Books.

Tinbergen, J. 1988. "Professor Tinbergen's Economics: A Comment on Dopfer." Journal of Economic Issues 22 (September): 851-54. 